\title{
Mathematical formulation to predict the harmonics of the superconducting Large Hadron Collider magnets
}

\author{
Nicholas Sammut \\ CERN-The European Organization for Nuclear Research, CH-1211 Geneva, Switzerland \\ and UOM-The University of Malta, Msida MSD 06, Malta \\ Luca Bottura \\ CERN-The European Organization for Nuclear Research, CH-1211 Geneva, Switzerland \\ Joseph Micallef \\ UOM-The University of Malta, Msida MSD 06, Malta \\ (Received 2 November 2005; published 9 January 2006)
}

\begin{abstract}
CERN is currently assembling the LHC (Large Hadron Collider) that will accelerate and bring in collision $7 \mathrm{TeV}$ protons for high energy physics. Such a superconducting magnet-based accelerator can be controlled only when the field errors of production and installation of all magnetic elements are known to the required accuracy. The ideal way to compensate the field errors obviously is to have direct diagnostics on the beam. For the LHC, however, a system solely based on beam feedback may be too demanding. The present baseline for the LHC control system hence requires an accurate forecast of the magnetic field and the multipole field errors to reduce the burden on the beam-based feedback. The field model is the core of this magnetic prediction system, that we call the field description for the LHC (FIDEL). The model will provide the forecast of the magnetic field at a given time, magnet operating current, magnet ramp rate, magnet temperature, and magnet powering history. The model is based on the identification and physical decomposition of the effects that contribute to the total field in the magnet aperture of the LHC dipoles. Each effect is quantified using data obtained from series measurements, and modeled theoretically or empirically depending on the complexity of the physical phenomena involved. This paper presents the developments of the new finely tuned magnetic field model and, using the data accumulated through series tests to date, evaluates its accuracy and predictive capabilities over a sector of the machine.
\end{abstract}

DOI: 10.1103/PhysRevSTAB.9.012402

PACS numbers: 85.70.Ay, 41.85.Lc, 07.55.Db

\section{INTRODUCTION}

In order to steer the magnet production and monitor that the field quality is within the required specifications, the LHC quality assurance plan foresees the field mapping at room temperature of all superconducting and resistive magnets. In addition, field mapping is performed in cryogenic conditions on a percentage of the superconducting magnets, typically $10 \%$ to $20 \%$ of the production depending on the magnet family. The aim of these tests is to obtain and maintain a good warm-cold correlation and to quantify effects relevant for the field quality in operating conditions that cannot be determined through correlations or inferred from other data. Examples are the amount of iron saturation at the nominal field levels at collision energy, or the field decay and snap back during particle injection and initial acceleration [1,2].

We plan to synthesize and consolidate the above information in the field description for the LHC (or FIDEL). FIDEL will consist of the large database of measured data, and a field model that will be used to interpolate and extrapolate the data to the operating conditions of the LHC. In this paper we describe the field model, in particular, which is a decomposition of the measured field errors and their deviations from the reference design values based on a separation of contributions from different physical origins. FIDEL is expected to hold for all magnet types and families, with the appropriate adaptations and to a different degree of detail. The description is going to be most detailed for the LHC dipoles, for which the largest set of warm and cold measurements is available. For this reason we will focus on this magnet family, the LHC dipoles [3], in the description and examples reported below, implicitly assuming that the reasoning holds also for the other magnet types (e.g., quadrupoles and higher order corrector magnets).

\section{PHYSICAL DECOMPOSITION}

The functions describing high energy particle beams can be assumed constant over a magnet length. A magnet is therefore accurately modeled by a $2 \mathrm{D}$ complex function that applies over an effective length, $L_{\text {eff }}$. We sometimes reduce the description to the integrated value of the field over the magnet length as done with lenses in classical optics.

In the following, we indicate with $\boldsymbol{C}_{\boldsymbol{n}}$ the complex harmonic of order $n$ in the complex series expansion of the 2D magnetic field $\boldsymbol{B}$ in the magnet aperture: 


$$
\begin{aligned}
\mathbf{B}(x, y) & =B_{y}+i B_{x}=\sum_{n=1}^{\infty} \mathbf{C}_{n}\left(\frac{\mathbf{z}}{R_{\mathrm{ref}}}\right)^{n-1} \\
& =\sum_{n=1}^{\infty}\left(B_{n}+i A_{n}\right)\left(\frac{x+i y}{R_{\mathrm{ref}}}\right)^{n-1}
\end{aligned}
$$

where $z$ is the coordinate in the complex plane and $R_{\text {ref }}$ is the reference radius. The coefficients $\boldsymbol{C}_{\boldsymbol{n}}$ have dimensions of $[\mathrm{T}]$.

The index $m$ stands for the order of the main field (with $m=1$ for dipole), so that the main field is indicated as $B_{m}$ (in $T$ at the reference radius $R_{\text {ref }}=17 \mathrm{~mm}$ ). The nonnormalized harmonic coefficients $\boldsymbol{C}_{\boldsymbol{n}}$ are assumed to be given in the reference frame aligned with the main field direction. They can be decomposed in their real part $B_{n}$ (the normal harmonics giving a vertical field component in the horizontal plane) and imaginary part $A_{n}$ (the skew harmonics), and, because we take the main field to be purely normal, we have by definition $A_{m}=0$. For convenience we use also normalized harmonic coefficients, indicated as $\mathbf{c}_{\mathbf{n}}$ and defined as

$$
\boldsymbol{c}_{\boldsymbol{n}}=b_{n}+i a_{n}=10^{4} \frac{\mathbf{C}_{n}}{B_{m}}
$$

expressed in [units], and also decomposed in their real part $b_{n}$ (the normal harmonics) and imaginary part $a_{n}$ (the skew harmonics). In practice the above normalization is useful only for the harmonics of order higher than the main field, i.e., for

$$
n \geq m+1 .
$$

Finally, the main field transfer function (TF) is defined as the ratio of field generated and operating current:

$$
T F=\frac{B_{m}}{I},
$$

which is expressed in units of $[T / A]$.

The field model for the main field and the higher order harmonics is the generic relation:

$$
\mathbf{C}_{n}=\mathbf{C}_{n}\left(t, I, \frac{d I}{d t}, T, I(-t)\right)
$$

where we express the fact that the harmonic $\boldsymbol{C}_{\boldsymbol{n}}$ depends on time $(t)$, magnet operating current $(I)$, magnet ramp rate $(d I / d t)$, magnet temperature $(T)$, and magnet powering history $I(-t)$. Note that above we have referred to the local value of the field along the magnet. We postulate and verify, however, that the model also applies to the field integral, the integral transfer function and the average higher order harmonics.

To give an explicit form of the field model, we decompose the field errors in the following components. The advantage of the decomposition relies on the fact that each component has a clear physical origin, and that it can be determined directly from field measurements:
(1) dc components $\left(\mathbf{C}_{n}^{\mathrm{dc}}\right)$. - which are steady state in nature. They are reproducible from cycle to cycle and depend only on current, while they do not depend on time or the ramp rate $(d I / d t)$.

a. Geometric contribution $\left(\mathbf{C}_{n}^{\text {geometric }}\right)$. - which is due to the deviation between the conductor placement in the real coil winding and the ideal distribution of current (i.e., producing the exact, desired multipolar field). This contribution is present at all field levels and is proportional to the operating current.

b. dc magnetization contribution $\left(\mathbf{C}_{n}^{M \mathrm{dc}}\right)$. - - originated by the persistent currents in the superconducting filaments. This contribution is important at low operating field (e.g., injection in the main dipoles), where the superconductor magnetization is highest. It is reproducible provided that the magnet is cycled according to the same procedure, in particular, maximum and minimum current, irrespective of the time required for cycling.

c. Saturation contribution $\left(\mathbf{C}_{n}^{\text {saturation }}\right)$. - which is due to changes of the magnetic permeability in the iron yoke surrounding the coils. This contribution is important at high field, and mainly affects the main field component.

d. Displacement contribution $\left(\mathbf{C}_{n}^{\text {deformation }}\right)$. — caused by displacements of the cables in the coil cross section. Cable movements can take place, for instance, during cool-down and powering at high field as a consequence of the changes in the force and stress distribution.

e. Residual magnetization contribution $\left(\mathbf{C}_{n}^{\text {residual }}\right)$. - of magnetic parts in the cold mass, mostly in the collars and iron surrounding the coils, visible at low current, e.g., during warm measurements.

(2) ac components $\left(\mathbf{C}_{n}^{\mathrm{ac}}\right)$. - which depend on current, time, ramp rate, and powering history and can be nonreproducible from one powering cycle to the following one.

a. Decay $\left(\mathbf{C}_{n}^{\text {decay }}\right)$. - an effect due to field changes internal to the cable [4] and, to a much lower extent, flux creep in the filaments magnetization. This effect is important during beam injection and in general whenever the current is kept constant at low field. The magnitude of the decay depends on the powering history, both on the waveform of the powering cycle as well as waiting times, and has memory of previous powering cycles, thus making this effect nonreproducible from cycle to cycle.

b. Snap back $\left(\mathbf{C}_{n}^{\text {snap back }}\right)$. - which is the rapid reestablishment of the magnetization after its decay during a constant current plateau, important at the beginning of the acceleration ramp. The same considerations on reproducibility are valid as for the decay.

c. Coupling currents $\left(\mathbf{C}_{n}^{M \mathrm{ac}}\right)$.- - which is the contribution due to interfilamentary currents within the strand and interstrand currents within the cable. This contribution is only present during changes in the operating field, e.g., during energy ramp. It is reproducible and depends on the ramp rate $(d I / d t)$. 

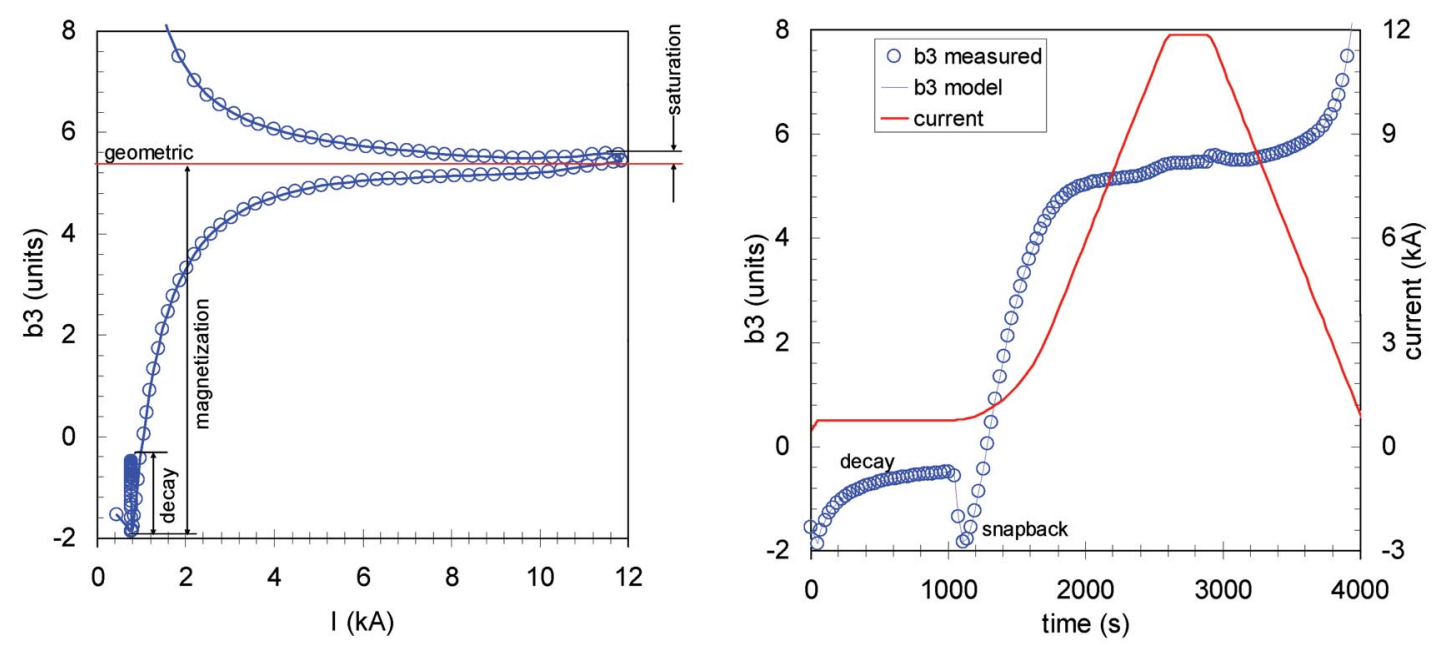

FIG. 1. (Color) Integral sextupole of typical dipole magnet plotted vs current (left) and vs time (right). Modeling is based on the expressions discussed in this paper. The right plot also reports the nominal excitation current during simulated LHC cycle (solid red line, right axis).

We report in Fig. 1 an example of the data that can be obtained from the cold test of an LHC dipole, plotting the dependence of the normal sextupole component (the first harmonic allowed in a dipole) as a function of current and field along a simulated LHC accelerator operating cycle. In the same plot we have also evidenced the various contributions to the total sextupole field error, in accordance to the list above. As described in Sec. II, an analytical interpolation is used to accurately model each contribution and, eventually, the measured data. The result of modeling is shown in Fig. 1 for the sole purpose of visual comparison with the measured data. We will discuss later in the paper the quality of the model proposed.

\section{MATHEMATICAL FORMULATION}

The field model relies on the linearity of the field error decomposition, i.e., on the mutual independence of the components. As described above, this is justified by the inherent different and independent physical origin of the components. Moreover, as a general rule, superconducting magnets (and especially the main dipoles and quadrupoles) are designed to achieve relative field errors of $0.1 \%$ or better.

Any coupling between the components which results from variations in the field distribution is at most 6 orders of magnitude smaller than the background field. This coupling effect is an order of magnitude smaller than the required modeling error (typically 0.1 units for $b_{3}$ which corresponds to 5 orders of magnitude smaller than the main field) and is therefore negligible.

In other words, all deviations from linearity are small perturbations of the ideal field, and the single contributions to the field errors can be added linearly to obtain the total field in the magnet.
Under this assumption, the field model can be given by the sum of the contributions

$$
\mathbf{C}_{n}=\mathbf{C}_{n}^{\mathrm{dc}}+\mathbf{C}_{n}^{\mathrm{ac}},
$$

where $\mathbf{C}_{n}^{\mathrm{dc}}$ is the dc component and $\mathbf{C}_{n}^{\mathrm{ac}}$ is the ac component which are defined as

$$
\begin{aligned}
\mathbf{C}_{n}^{\mathrm{dc}}= & \mathbf{C}_{n}^{\text {residual }}+\mathbf{C}_{n}^{\text {geometric }}+\mathbf{C}_{n}^{\text {deformation }}+\mathbf{C}_{n}^{M \mathrm{dc}} \\
& +\mathbf{C}_{n}^{\text {saturation }}, \\
& \mathbf{C}_{n}^{\mathrm{ac}}=\mathbf{C}_{n}^{\text {decay }}+\mathbf{C}_{n}^{\text {snap-back }}+\mathbf{C}_{n}^{M \mathrm{ac}} .
\end{aligned}
$$

In the following sections, we give analytical formulas suitable to describe the various dependencies, with a limited set of free parameters.

\section{A. Geometric contributions}

The geometric contributions to the field and field errors is proportional to the excitation current in the magnet. Hence, the geometric contribution may be written as

$$
B_{m}^{\text {geometric }}=\gamma_{m} I
$$

where $I$ is the excitation current in the magnet. The geometric coefficient, $\gamma_{m}$, in the above definition includes the linear contribution from the iron yoke thus ignoring the saturation and the residual magnetization. For the transfer function:

$$
T F^{\text {geometric }}=\gamma_{m}
$$

and for the normalized harmonics:

$$
\mathbf{c}_{n}^{\text {geometric }}=\gamma_{n}
$$

The geometric contribution is the only component of the model that can be obtained through extrapolation from 
warm measurements [5] using the equations

$$
B_{m}^{\text {geometric }}=g_{m}^{w c} B_{m}^{\text {warm }}+\Delta_{m}^{w c},
$$

where $g_{m}^{w c}$ is the correlation gradient, $B_{m}^{\text {warm }}$ is the main field measured at warm and $\Delta_{m}^{w c}$ is the $y$ intercept of the correlation line. For the transfer function:

$$
T F^{\text {geometric }}=g_{m}^{w c} T F^{\mathrm{warm}}+\frac{\Delta_{m}^{w c}}{I}
$$

and for the normalized harmonics:

$$
c_{n}^{\text {geometric }}=g_{n}^{w c} c_{n}^{\text {warm }}+\Delta_{n}^{w c} .
$$

We remark here that Eq. (14) is general, and holds both for local and integral harmonics, in which case it contains the contribution of the change in the magnetic length.

\section{B. dc magnetization contributions}

When the background field is varied during the field ramp, the superconducting filaments in a strand become magnetized. The magnetization $M$ is generated by persistent currents trapped in the filaments [6,7]. Hence, in first approximation, the magnetization is proportional to the critical current density $J_{c}$ and the filament diameter $D_{\text {fil }}$ [8]:

$$
M \propto J_{c} D_{\text {fil }} .
$$

The critical current density changes with field according to a law of the type [9]:

$$
J_{c} \propto \frac{1}{B}\left(\frac{B}{B_{c}(T)}\right)^{p}\left(1-\frac{B}{B_{c}(T)}\right)^{q}\left[1-\left(\frac{T}{T_{\mathrm{co}}}\right)^{1.7}\right]^{m},
$$

where $B$ is the background field, $B_{c}$ is the critical field of the material (14.5 for NbTi), $T$ is the temperature, $T_{\mathrm{co}}$ is the critical temperature ( $9.5 \mathrm{~K}$ for $\mathrm{NbTi})$, and $p, q$, and $m$ are pinning exponents that are typically in the range $p=0.5$, $q=1$, and $m=2$ for the NbTi alloy used in the LHC cables.

$M$ is stationary in time (dc) since the persistent currents have infinitely long time constants. Hence, the dc magnetization appears as a hysteretic contribution to the field and field errors that depends on the strength of the magnetization as well as on the geometric distribution of the magnetization vectors in the winding cross section. The strength of the magnetization decreases at increasing background field, through the $J_{c}$ dependence of Eq. (15). As a result the magnetization contribution is important at low field, i.e., injection level, where the magnitude and the variation of $M$ in the coil cross section is the largest. At high field level the contribution of the persistent current magnetization decreases rapidly in accordance with Eq. (16).

To provide a scaling for the field generated by the dc magnetization we use the expression for $J_{c}$ in Eq. (16). Current is substituted for field giving

$$
B_{m}^{M \mathrm{dc}}=\mu_{m} I_{\mathrm{inj}}\left(\frac{I}{I_{\mathrm{inj}}}\right)^{p_{m}}\left(\frac{I_{c}-I}{I_{c}-I_{\mathrm{inj}}}\right)^{q_{m}}\left(\frac{T_{\mathrm{co}}^{1.7}-T^{1.7}}{T_{\mathrm{co}}^{1.7}-T_{\text {meas }}^{1.7}}\right)^{m_{m}},
$$

where the injection current $I_{\mathrm{inj}}$ and the measurement temperature $T_{\text {meas }}$ are introduced as reference points so that the product of the three terms in $I$ is equal to 1 at $I_{\mathrm{inj}}$, and the term in $T$ is equal to 1 at $T_{\text {meas }}$. With this normalization, the value of $\mu_{m}$ can be interpreted as the value of the contribution of the dc magnetization to the total field measured at injection, which is at present a quantity directly stored in the measurement database. By writing Eq. (16) we make the assumption that the complex convolution of the distribution of magnetization vectors can be condensed in the fitting exponents $p_{m}, q_{m}$, and $m_{m}$. Dividing Eq. (17) by $I$ we obtain the contribution to the transfer function

$$
T F^{M \mathrm{dc}}=\mu_{m} \frac{I_{\mathrm{inj}}}{I}\left(\frac{I}{I_{\mathrm{inj}}}\right)^{p_{m}}\left(\frac{I_{c}-I}{I_{c}-I_{\mathrm{inj}}}\right)^{q_{m}}\left(\frac{T_{c o}^{1.7}-T^{1.7}}{T_{c o}^{1.7}-T_{\text {meas }}^{1.7}}\right)^{m_{m}},
$$

and the normalized harmonics originated by the dc magnetization are

$$
\mathbf{c}_{n}^{M \mathrm{dc}}=\mu_{n}\left(\frac{I_{\mathrm{inj}}}{I}\right)^{2-p_{n}}\left(\frac{I_{c}-I}{I_{c}-I_{\mathrm{inj}}}\right)^{q_{n}}\left(\frac{T_{\mathrm{co}}^{1.7}-T^{1.7}}{T_{\mathrm{co}}^{1.7}-T_{\text {meas }}^{1.7}}\right)^{m_{n}},
$$

which has a different form from Eq. (16) because of the renormalization to make $\mu_{n}$ the measured dc magnetization harmonic at injection.

For a monotonous ramp (ramp up or ramp down), the Eqs. (17)-(19) hold when the filaments in the coil are in a fully penetrated state, i.e., after the crossing of the hysteresis cycle (penetration phase). The expressions are the same for different ramp directions, but the coefficients $\mu_{m}$ and $\mu_{n}$ for a downward ramp have opposite sign (and approximately same value) to those that best fit an upward ramp.

\section{Iron saturation contribution}

The iron saturation contributions to the main field and field errors depend mostly on the iron yoke configuration and on the B-H characteristics of the iron structure. The iron yoke saturation appears as a nonlinearity of the field and the field errors with respect to the operating current. This deviation is especially visible at high field levels, when the extent of saturation becomes significant.

It is not easy to establish an a priori fit which can take both effects into account by simple parametrization of the magnet cross section. Therefore the choice is to fit the saturation contribution as a sum of rounded step functions. We have found that the following function, also plotted in Fig. 2, can be used to model the saturation with good 


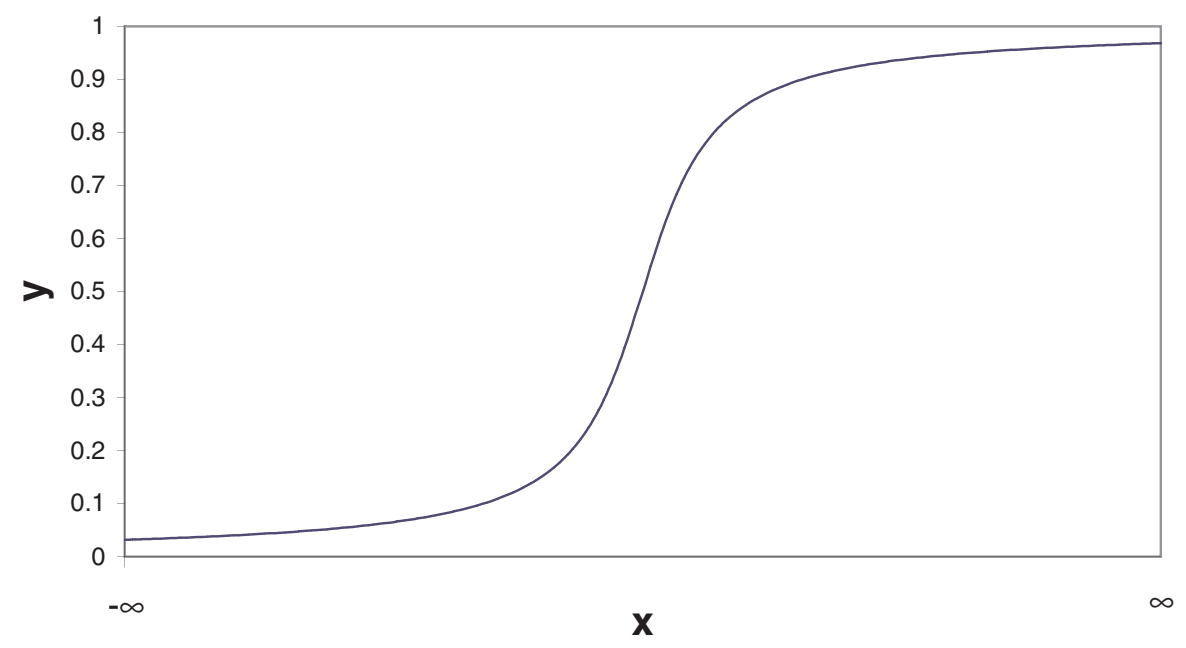

FIG. 2. (Color) Smooth step function $\left[y=\frac{1}{\pi} \operatorname{atan}(x)+\frac{1}{2}\right]$ used to describe the change in field due to saturation.

accuracy:

$$
\Sigma\left(I, S, I_{0}, I_{\text {nom }}\right)=\frac{1}{\pi} a \tan \left(S\left(\frac{I-I_{0}}{I_{\text {nom }}}\right)\right)+\frac{1}{2} .
$$

The main field is described as

$$
B_{m}^{\text {saturation }}=\sum_{i=1}^{N} \sigma_{m}^{i} I \Sigma\left(I, S_{m}^{i}, I_{0 m}^{i}, I_{\mathrm{nom}}\right) .
$$

$N$ is typically 1 or 2 depending on the complexity of the geometry of the iron yoke. The nominal current $I_{\text {nom }}$ is used to normalize the equation and $\sigma, S$, and $I_{0}$ are the fitting parameters (omitting subscripts and superscripts).

The transfer function is correspondingly

$$
T F^{\text {saturation }}=\sum_{i=1}^{N} \sigma_{m}^{i} \Sigma\left(I, S_{m}^{i}, I_{0 m}^{i}, I_{\text {nom }}\right)
$$

and the harmonic coefficients are

$$
c_{n}^{\text {saturation }}=\sum_{i=1}^{N} \sigma_{n}^{i} \Sigma\left(I, S_{n}^{i}, I_{0 n}^{i}, I_{\text {nom }}\right) .
$$

\section{Displacement contributions}

Contributions to field and field errors due to coil deformation under electromagnetic loads are proportional to the Lorentz forces, and, in case the constraints do not change during powering, these errors are proportional to the square of the current. In the most general situation, however, complex situations of establishment or loss of contact may arise. The contacts may be established or not depending on the amount of prestress in the structure. In general, the changes in field and field errors can only be obtained in detail using simulation codes that take into account the actual deformation for the specific preload case and therefore reconstruct the change in the field. In practice, we have found that the effect of the coil movement in the LHC magnets is small [10]. We have therefore decided to simplify the model, including any effect in the saturation formulation above.

\section{E. Residual magnetization contributions}

After powering at nominal current, the magnetic components (e.g., the iron yoke) can be permanently magnetized. The residual magnetization appears as a field contribution that is particularly important at small excitation currents, e.g., in warm conditions. The contribution to the main field can be written as

$$
B_{m}^{\text {residual }}=\rho_{m} I\left(\frac{I_{\text {inj }}}{I}\right)^{r_{m}}
$$

for the transfer function

$$
T F^{\text {residual }}=\rho_{m}\left(\frac{I_{\mathrm{inj}}}{I}\right)^{r_{m}}
$$

and for the field errors

$$
\mathbf{c}_{n}^{\text {residual }}=\rho_{n}\left(\frac{I_{\mathrm{inj}}}{I}\right)^{r_{n}} .
$$

\section{F. Decay}

The decay effect was discovered at the commissioning of the Tevatron (Fermilab-Illinois) in 1987 [11] where it caused the chromaticity to change by as much as 70 units over a few hours. It has been shown [12] that the decay of harmonics at constant current is driven by field changes on the strands caused by current redistribution in the superconducting cables. The amplitude of the current distribution process can be modeled by a diffusion equation, whose most general solution is a series of harmonics in space modulated by an exponential dependence in time. The decay phenomenon is quite complex: the current redistribution causes a change of the local field in the strands by few $\mathrm{mT}$, which in turn changes the persistent currents distribu- 


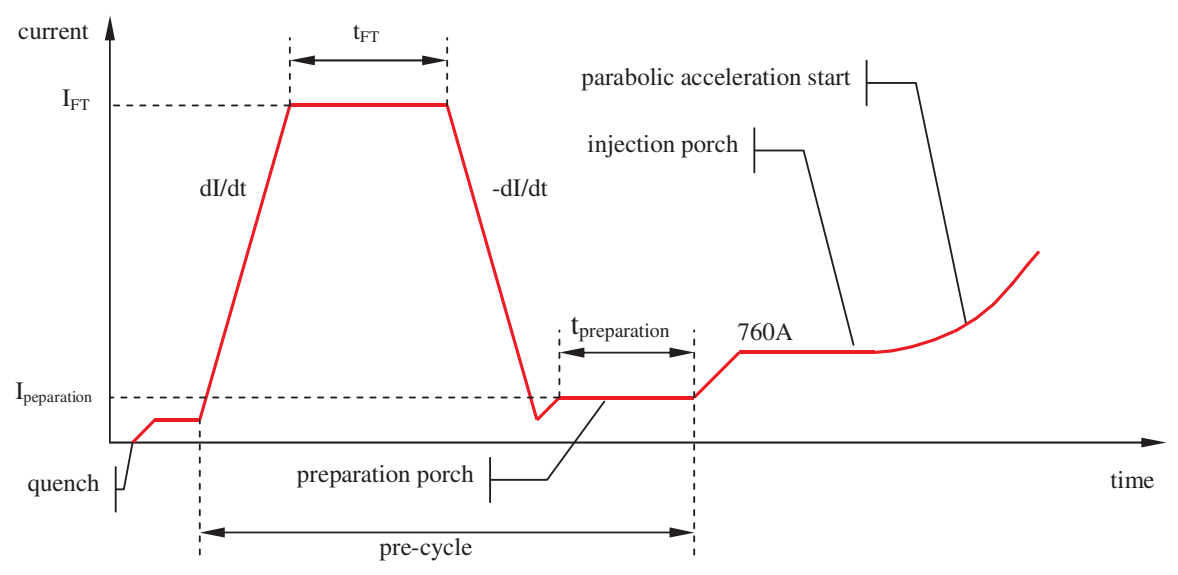

FIG. 3. (Color) Definition of the parameters affecting decay during LHC injection.

tion and the dc magnetization of the filaments by adding an arbitrary component to the initial magnetization state. This results in a net decrease of the average dc magnetization of the cables and an overall decrease of its contribution to the total field. Neglecting all nonlinearities, we make here the simplifying assumption that the dynamics of the field follows that of the current diffusion. The evolution follows the function [12]:

$$
\begin{aligned}
\Delta\left(t, t_{\mathrm{inj}}, \tau, a^{\Delta}\right)= & d\left(1-e^{-\left[\left(t-t_{\mathrm{inj}}\right) / \tau\right]}\right) \\
& +(1-d)\left(1-e^{-\left[\left(t-t_{\mathrm{inj}}\right) / 9 \tau\right]}\right),
\end{aligned}
$$

which holds for $I=I_{\mathrm{inj}}$ and $t>t_{\mathrm{inj}} . t$ is the instantaneous time, $t_{\text {inj }}$ is the time at injection, $I_{\text {inj }}$ is the current at injection, $\tau$ is the time constant. The parameter $d$ gives the normalized weight of the fast component of the decay and its complement to one, $1-d$, gives the normalized weight of the slow component. The field decay, using Eq. (27), is given by

$$
B_{m}^{\mathrm{decay}}=\delta_{m} I \frac{\Delta\left(t, t_{\mathrm{inj}}, \tau_{m}, d_{m}\right)}{\Delta\left(t_{\mathrm{inj}}^{s t d}, t_{\mathrm{inj}}, \tau_{m}, d_{m}\right)},
$$

where the parameter $\delta_{m}$ represents the decay at the end of an injection lasting a reference time $t_{\mathrm{inj}}^{\text {std }}$. The contribution of decay to the transfer function is modeled by

$$
T F^{\text {decay }}=\delta_{m} \frac{\Delta\left(t, t_{\mathrm{inj}}, \tau_{m}, d_{m}\right)}{\Delta\left(t_{i n j}^{s t d}, t_{\mathrm{inj}}, \tau_{m}, d_{m}\right)},
$$

and by analogy the contribution to the harmonics is given by

$$
\mathbf{c}_{n}^{\text {decay }}=\delta_{n} \frac{\Delta\left(t, t_{\mathrm{inj}}, \tau_{n}, d_{n}\right)}{\Delta\left(t_{i n j}^{s t d}, t_{\mathrm{inj}}, \tau_{n}, d_{n}\right)} .
$$

The amount of decay depends mostly on the powering history (Fig. 3). In practice the powering history can be condensed in a single powering cycle characterized by the current reached at the flattop $I_{F T}$, the flattop duration $t_{F T}$, the time $t_{\text {preparation }}$ elapsed (ramp down, preparation, ramp to injection) between the end of the flattop and the injection.

The scaling for the decay amplitudes (we omit the subscripts $m$ and $n$ for simplicity) are as follows:

$$
\delta=\left(E \frac{I_{F T}}{I_{F T}^{s t d}}\right)\left(\frac{T_{0}-T_{1} e^{-\left(t_{F T} / \tau_{T}\right)}}{T_{0}-T_{1} e^{-\left(t_{F T}^{s t d} / \tau_{T}\right)}}\right)\left(\frac{P_{0}+P_{1} e^{-\left(t_{\text {preparation }} / \tau_{P}\right)}}{P_{0}+P_{1} e^{-\left(t_{\text {preparation }}^{s t d} / \tau_{P}\right)}}\right),
$$

where $I_{F T}^{s t d}, t_{F T}^{s t d}, t_{\text {preparation }}^{\text {std }}$ are the normalization parameters and $E, T_{0}, T_{1}, \tau_{T}, P_{0}, P_{1}, \tau_{P}$ are the fitting parameters.

\section{G. Snap back}

The snap back phenomena was discovered two years after the decay at the Tevatron (Fermilab-Illinois) [13]. During snap back the field bounces back to its predecay level once the current in the magnet starts to ramp up after a stop, e.g., at injection. Fast sextupole measurements in the LHC and Tevatron main bending dipole magnets have shown that the sextupole snap back can be described well (within a standard deviation of 0.02 units) by an exponential fitting of the type [14]:

$$
b_{3}^{\text {snap back }}(t)=b_{3}^{\text {decay }} e^{-\frac{I(t)-I_{\text {injection }}}{\Delta I}}
$$

where $b_{3}^{\text {snap back }}(t)$ is the sextupole change during the snap back, $I(t)$ is the instantaneous value of the current, initially at the injection value $I_{\text {inj. }}$. The snap-back amplitude $b_{3}^{\text {decay }}$ and the current change $\Delta I$ are the two fitting constants. In addition, the fitting parameters are strongly correlated, and once represented in a scatter plot $\Delta b_{3}$ vs $\Delta I$ they lie on a straight line [14]:

$$
\Delta b_{3}=g_{3}^{S B} \Delta I,
$$

where $g_{3}^{S B}$ is a constant that can be determined using measurements on a sample of the magnet population. Based on this observation the snap back can be modeled 
by an expression of the type given above so that

$$
\begin{gathered}
B_{m}^{\text {snap back }}=B_{m}^{\text {decay }}\left(t_{\text {ramp }}\right) e^{-\left[\left(I(t)-I_{\text {inj }}\right) /\left(\Delta I_{m}\right)\right]}, \\
T F^{\text {snap back }}=T F^{\text {decay }}\left(t_{\text {ramp }}\right) e^{-\left[I(t)-I_{\text {inj }}\right] / \Delta I_{m}}, \\
\mathbf{c}_{n}^{\text {snap back }}=\mathbf{c}_{n}^{\text {decay }}\left(t_{\text {ramp }}\right) e^{-\frac{I(t)-I_{\text {inj }}}{\Delta I_{n}}},
\end{gathered}
$$

where the factors $B_{m}^{\text {decay }}\left(t_{\text {ramp }}\right), T F^{\text {decay }}\left(t_{\text {ramp }}\right)$, and $c_{n}^{\text {decay }}\left(t_{\text {ramp }}\right)$ are, respectively, the change of the main field, the transfer function and the normalized harmonics during the decay, evaluated at the time of the start of the ramp $t_{\text {ramp }}$. The characteristic current changes for the main field $\Delta I_{m}$ and for the normalized harmonics $\Delta I_{n}$ are given by

$$
\begin{gathered}
\Delta I_{m}=\frac{B_{m}^{\text {decay }}}{g_{m}^{S B}}, \\
\Delta I_{n}=\frac{c_{n}^{\text {decay }}}{g_{n}^{S B}} .
\end{gathered}
$$

\section{H. Coupling currents contributions}

Eddy currents are induced in loops among the transposed superconducting filaments in the strands, or among the strands in the cables. These currents couple the filaments and strands electromagnetically and are often referred to as coupling currents. They have time constants in the range of few milliseconds (among filaments in the strands) to few hundreds of milliseconds (among strands in cables). Therefore, for the typical ramp times to be used in the LHC operation, they can be assumed to be fully developed in the resistive regime, that is all inductive and shielding effects have already decayed. We also neglect the field dependence of the total resistance of the coupling current loops.

With this assumption, the contribution of coupling currents to main field and field errors is linear with the ramp rate. We write therefore for the main field component that

$$
B_{m}^{M \mathrm{ac}}=\theta_{m} I_{\mathrm{inj}} \frac{1}{10} \frac{d I}{d t}
$$

where the normalization factor is used to refer the contribution to the nominal ramp rate of the LHC $(10 \mathrm{~A} / \mathrm{s})$. The contribution to the transfer function is

$$
T F^{M \mathrm{ac}}=\theta_{m} \frac{I_{\mathrm{inj}}}{I} \frac{1}{10} \frac{d I}{d t},
$$

TABLE I. Summary of the fitting parameters contained in the field model.

\begin{tabular}{lcccc}
\hline \hline & & & Units & \\
Symbol & Meaning & $B_{m}$ & TF & $\mathbf{c}_{\mathbf{n}}$ \\
\hline$\gamma$ & Geometric field error & $(T / A)$ & $\left(T_{f} / A\right)$ & (Units) \\
$g^{w c}$ & Warm-cold correlation gradient & $(-)$ & $(-)$ & $(-)$ \\
$\Delta^{w c}$ & Warm-cold correlation intercept & $(T)$ & $(T)$ & (Units) \\
$\mu$ & dc magnetization strength & $(T / A)$ & $(T / A)$ & (Units) \\
$p$ & dc magnetization pinning exponent & $(-)$ & $(-)$ & $(-)$ \\
$q$ & dc magnetization pinning exponent & $(-)$ & $(-)$ & $(-)$ \\
$m$ & dc magnetization pinning exponent & $(-)$ & $(-)$ & $(-)$ \\
$\sigma$ & Iron saturation strength & $(T / A)$ & $\left(T_{f} / A\right)$ & $($ Units) \\
$I_{0}$ & Iron saturation curre & $(A)$ & $(A)$ & $(A)$ \\
$S$ & Iron saturation current range & $(-)$ & $(-)$ & $(-)$ \\
$\rho$ & Residual magnetization strength & $(T / A)$ & $(T / A)$ & $($ Units $)$ \\
$r$ & Residual magnetization exponent & $(-)$ & $(-)$ & $(-)$ \\
$\delta$ & Decay strength & $(T / A)$ & $(T / A)$ & $($ Units) \\
$d$ & Fast decay normalized amplitude & $(-)$ & $(-)$ & $(-)$ \\
$\tau$ & decay time constant & $(\mathrm{s})$ & $(\mathrm{s})$ & $(\mathrm{s})$ \\
$E$ & flattop current dependence of decay & $(-)$ & $(-)$ & $(-)$ \\
$T_{0}$ & flattop time dependence of decay & $(-)$ & $(-)$ & $(-)$ \\
$T_{1}$ & flattop time dependence of decay & $(-)$ & $(-)$ & $(-)$ \\
$\tau_{T}$ & flattop time decay time constant & $(\mathrm{s})$ & $(\mathrm{s})$ & $(\mathrm{s})$ \\
$P_{0}$ & Preparation time dependence of decay & $(-)$ & $(-)$ & $(-)$ \\
$P_{1}$ & Preparation time dependence of decay & $(-)$ & $(-)$ & $(-)$ \\
$\tau_{P}$ & Preparation time decay time constant & $(\mathrm{s})$ & $(\mathrm{s})$ & $(\mathrm{s})$ \\
$g^{S B}$ & Snap-back correlation gradient & $(T / A)$ & $N / A$ & $($ Units/A) \\
$\theta$ & Coupling currents strength & $(T / A)$ & $(T / A)$ & $($ Units) \\
\hline \hline & & & &
\end{tabular}



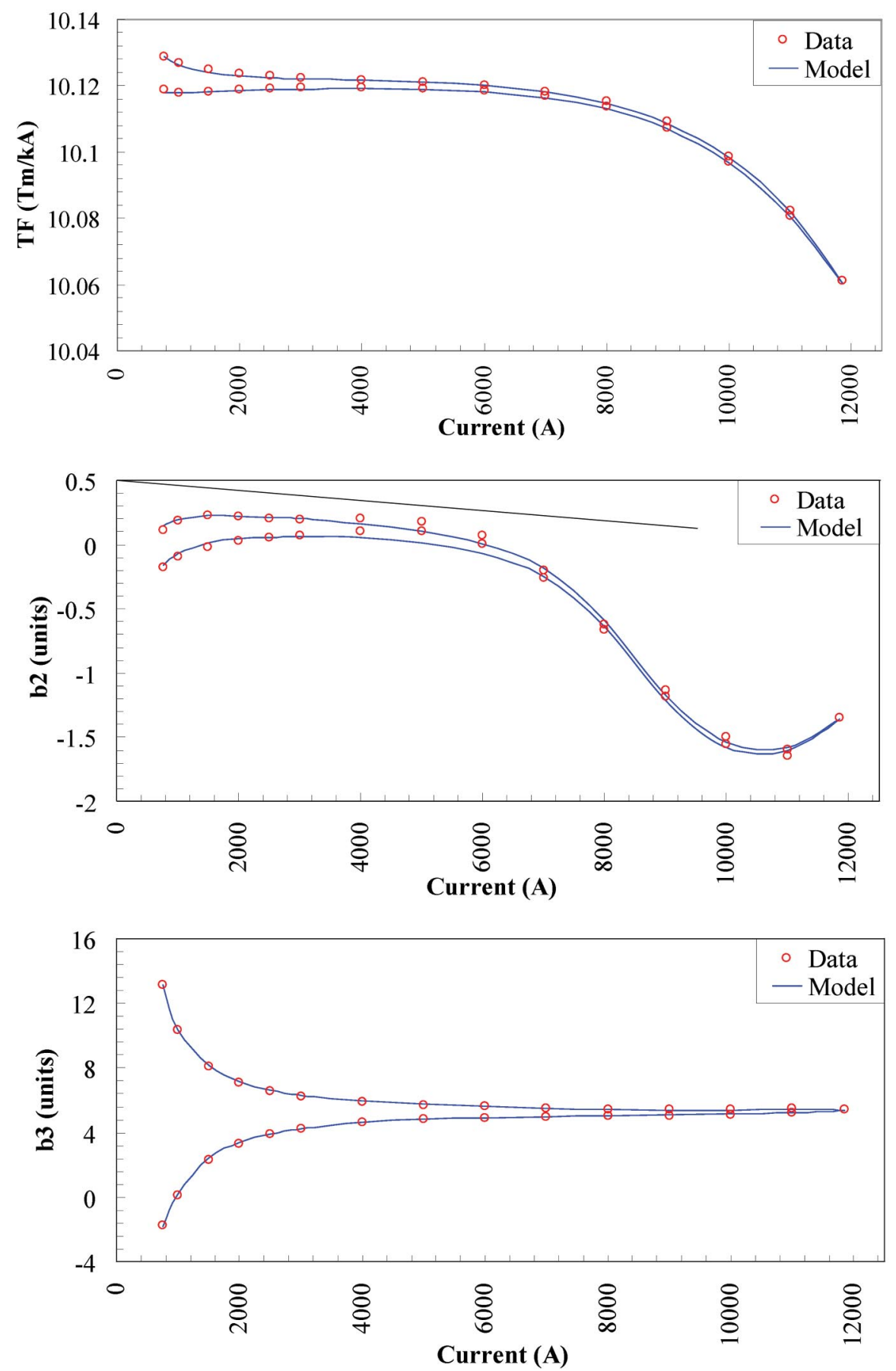

FIG. 4. (Color) Modeling of the integral transfer function (TF), the normal quadrupole and the normal sextupole using load line data from all cold tested magnets in the LHC sector 7-8.

while for the normalized harmonics

$$
\mathbf{c}_{n}^{M \mathrm{ac}}=\theta_{n} \frac{I_{\mathrm{inj}}}{I} \frac{1}{10} \frac{d I}{d t}
$$

Note that normalization is such that the multiplication constant corresponds to the effect of the coupling currents at injection current and nominal ramp rate in both cases.

\section{Summary of parameters}

The expressions discussed in the previous sections contain several fitting parameters with different meanings and units. To maintain a good overview, we report in Table I a summary of the 24 fitting parameters contained in the field model as devised here. Subscripts (for main field, transfer function and higher order harmonics) and superscripts (for summation indices) have been omitted for clarity. All 

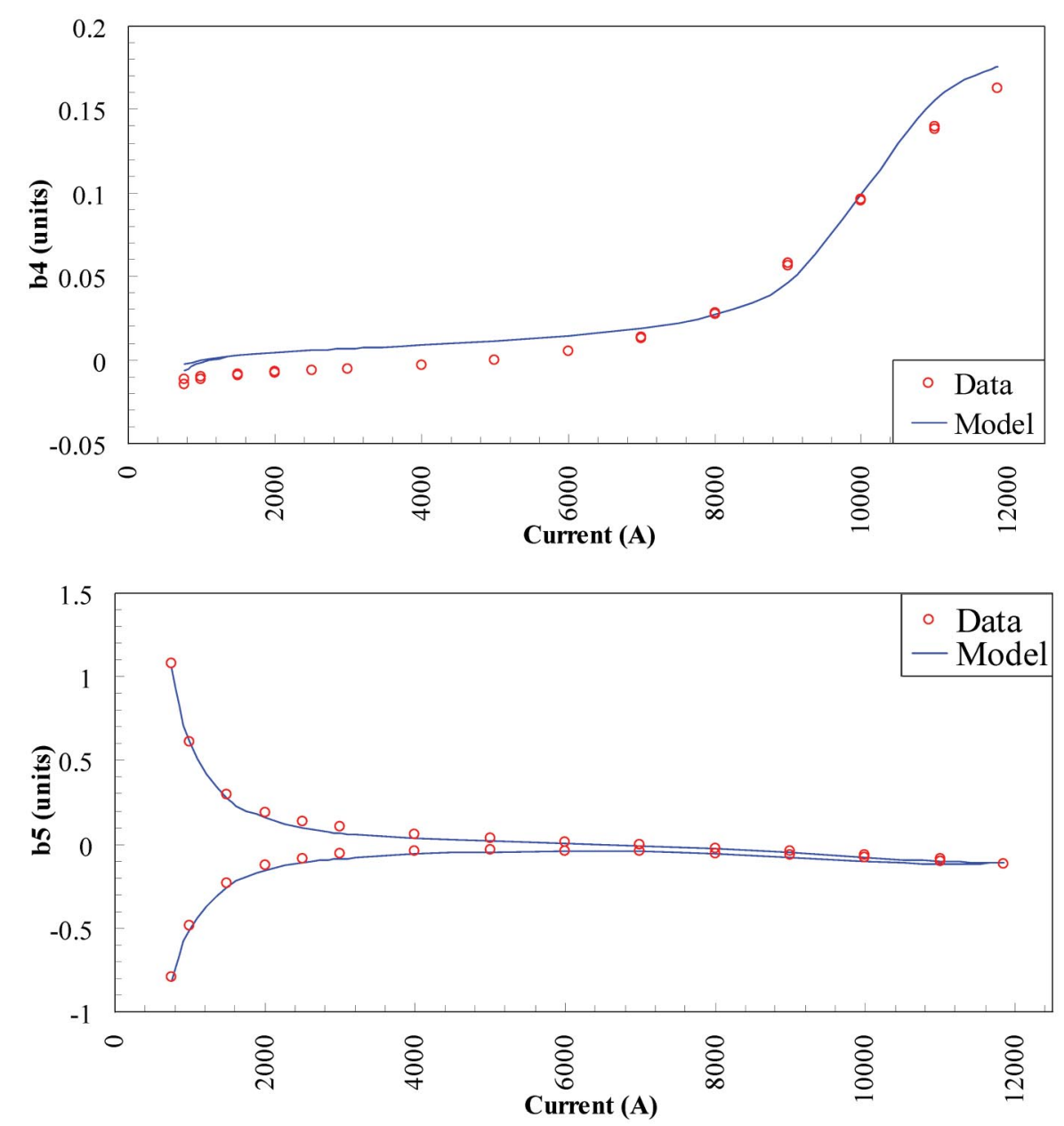

FIG. 5. (Color) Modeling of the normal octupole and normal decapole using load line data from all cold tested magnets in the LHC sector 7-8.

parameters can be determined univocally from measured data, as shown in the following section.

\section{EXAMPLES OF APPLICATION}

To give a flavor for the capability of the field model, we present here as an example of application the forecast of the field and field errors in a sector $(1 / 8)$ of the LHC during a standard operating cycle. We used the data collected during the cold test of the magnets to be installed in sector 7-8. The data was taken on 65 magnets (130 apertures) along the magnet load line in a series of constant current steps, and is therefore relevant only for the dc field quality. The measured field and field quality was averaged over the magnets of the sector to obtain an estimate of the integral quantities. These were finally modeled using the dc error components, i.e., the geometric contribution, the dc magnetization from persistent currents, the iron saturation and displacement contribution, and the residual magnetization.

The results for the main field transfer function and the first harmonics are reported in Figs. 4-6. All major fea- tures of the current dependence are reproduced very well. Figure 7 reports the model error defined as the maximum deviation between the analytical model and the measured data. The model is quite effective, providing an interpolation of the field and field quality to better than 0.1 units @ $17 \mathrm{~mm}$ for the harmonics and 0.44 units for the TF in the range between injection $(760 \mathrm{~A})$ and collision current $(11850 \mathrm{~A})$. The set of parameters used for the modeling is reported in Table II.

The parameters in Table II have been grouped according to the physical origin. Because of the definitions discussed in this paper, the parameters in bold also represent the order of magnitude of the contribution and give a direct indication of the relative weight of each component. As expected, and depending on the harmonic considered some components are dominant. This is consistent with the curves shown in Fig. 4. For instance, in the case of the $\mathrm{TF}$ and $b_{2}$, the saturation component is dominant over the $\mathrm{dc}$ and the residual magnetization by 2 orders of magnitude. Therefore, the modeling of the saturation in this case is very important (effect of 60 units of TF and 2 units of 

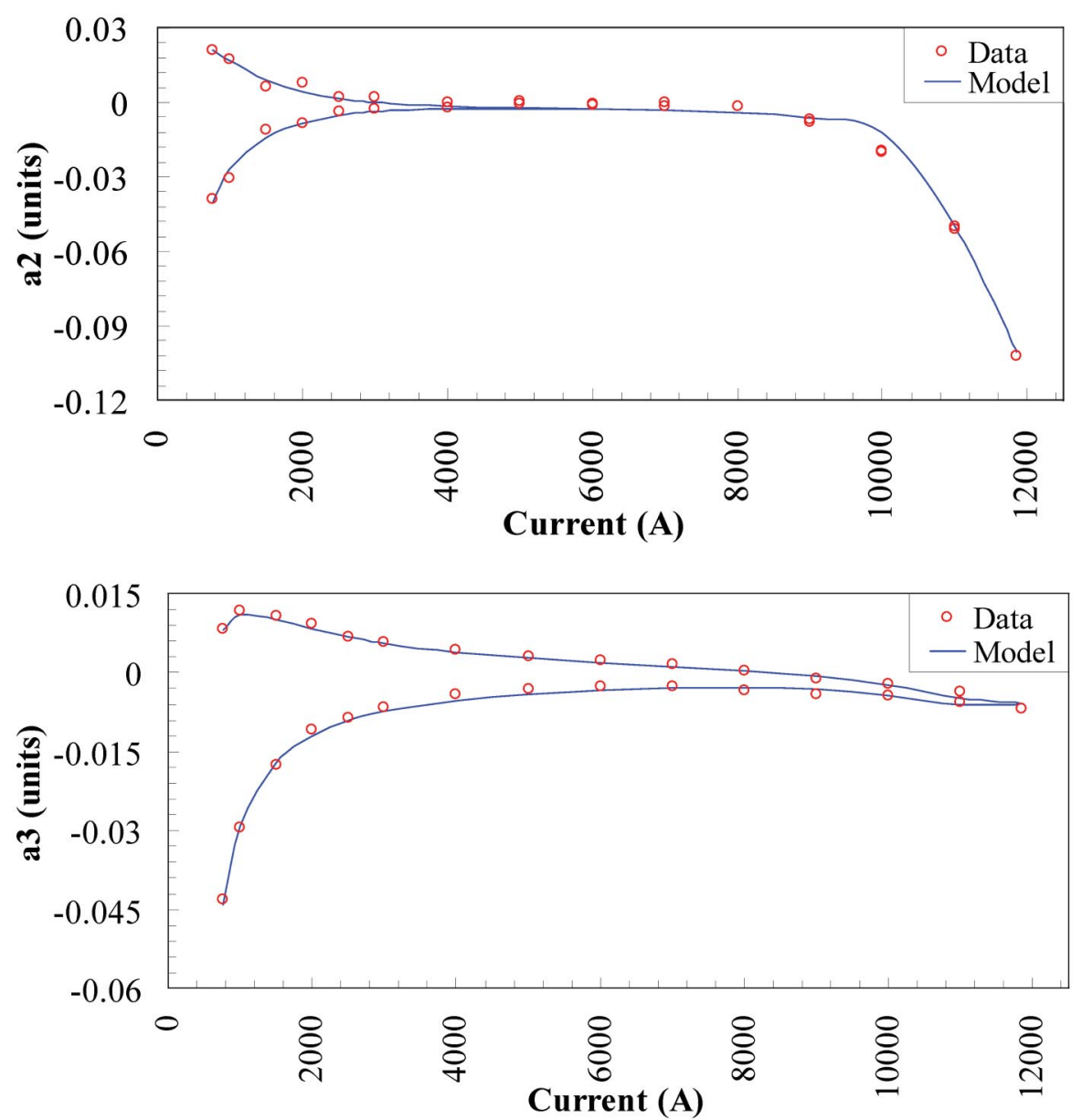

FIG. 6. (Color) Modeling of the skew quadrupole and skew sextupole using load line data from all cold tested magnets in the LHC sector 7-8.

$b_{2}$ ), while the modeling of the other components is less critical (effect of 6 units of TF and 0.2 of $b_{2}$ ). By contrast, in the case of $b_{3}$, the geometric and dc magnetization are the dominant components (effect of 7 units) while the saturation and the residual magnetization modeling can be regarded as a fine tuning correction (effect of 0.3 units).
We note here that the results presented in Fig. 7 are only a part of the overall modeling error. The main additional error source will be the model of the ac components (decay and snap back), and, in particular, the powering history dependence of Eq. (31), and the snap-back model Eqs. (34)-(36). We are presently in the process of testing the scaling properties of the above expressions [14]. An

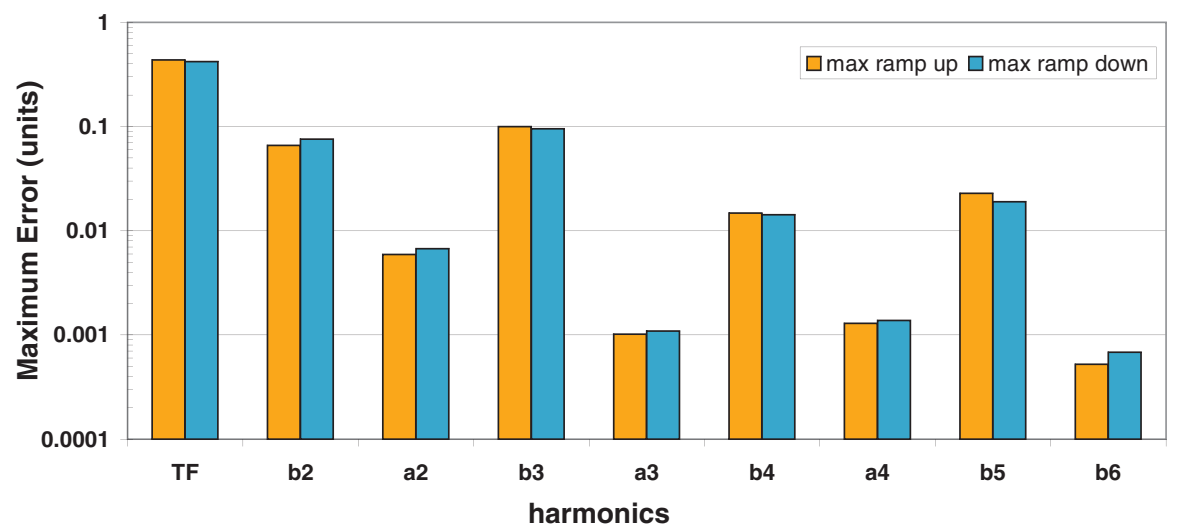

FIG. 7. (Color) Maximum error between measured data and model, derived from the plots of Figs. 4-6. (Note that the TF plot of Fig. 4 is plotted in Tm/kA while the TF error here is in units to maintain consistency.) 
TABLE II. Parameters used for modeling the field and field errors in the LHC sector 7-8. The units of the parameters are the same as shown in Table I.

\begin{tabular}{|c|c|c|c|c|c|c|c|c|}
\hline \multirow[t]{2}{*}{ Coefficient } & \multicolumn{7}{|c|}{ Value } & \multirow[t]{2}{*}{ Component } \\
\hline & $\mathrm{TF}$ & $\mathrm{b} 2$ & $\mathrm{a} 2$ & b3 & a3 & b4 & b5 & \\
\hline$\gamma$ & 10.119 & 0.142 & -0.040 & 5.276 & -0.236 & 0.004 & 0.245 & Geometric \\
\hline$\mu$ & -0.005 & 0.154 & -0.031 & -7.466 & 0.026 & -0.002 & 0.931 & \multirow{4}{*}{ dc magnetization } \\
\hline$p$ & 1.11 & 1.54 & 1.46 & 0.63 & 1.11 & -1.28 & 0.12 & \\
\hline$q$ & -0.29 & 0.96 & 11.52 & 0.55 & 0.98 & 0.57 & -0.39 & \\
\hline$m$ & 2 & 2 & 2 & 2 & 2 & 2 & 2 & \\
\hline$\sigma^{1}$ & 0.247 & -3.241 & -0.118 & -0.095 & -0.008 & 0.207 & -0.142 & \multirow{6}{*}{ Saturation } \\
\hline$I_{0}{ }^{1}$ & 10739 & 8569 & 11090 & 7224 & 10256 & 10056 & 9214 & \\
\hline$S^{1}$ & 1.691 & 8.088 & 32.181 & 9.760 & 10.453 & 12.985 & 8.150 & \\
\hline$\sigma^{2}$ & -0.545 & 20.131 & & 0.347 & & & & \\
\hline$I_{0}^{2}$ & 13599 & 14107 & & 11031 & & & & \\
\hline$S^{2}$ & 3.230 & 25.551 & & 16.923 & & & & \\
\hline$\rho$ & 0.003 & -0.182 & -0.008 & 0.340 & -0.018 & -0.011 & 0.126 & \multirow{2}{*}{ Residual magnetization } \\
\hline$r$ & 1.86 & 1.95 & 2.82 & 10.00 & 2.52 & 1.36 & 2.85 & \\
\hline
\end{tabular}

additional source of uncertainty will be the changes in the geometry of the magnets themselves, following powering and thermal cycles. These changes are small and difficult to describe in a predictive, analytical model, and we have therefore chosen to neglect them.

\section{CONCLUSION}

We have described in detail the elements of a field model that can provide an accurate forecast of current ramps and corrections for the LHC control. The field model is based on a number of components that can be individually identified and physically decomposed to model the total field in the magnet aperture of the magnets. This technique was implemented using the data of series cold measurements of a sample of the LHC dipoles and yields very encouraging results. The dc field can be interpolated to 0.44 units for the TF and to better than 0.1 units @ $17 \mathrm{~mm}$ for the low order field errors, which is fully satisfactory. Further tests are mandatory, and planned, to asses the prediction and modeling capability of injection decay and snap back.

The main advantage of the model described here is that each component has a clear correspondence to a physical origin, while the parametrization chosen still allows large flexibility. This is, in particular, important to refine the model iteratively based on the results of first beam measurements, expected in 2007 , as well as special measurement campaigns on spare and leftover magnets.

[1] Louis Walckiers, Luca Bottura, Marco Buzio, Stephane Fartoukh, Stephan Russenschuk, Stephane Sanfilippo,
Walter Scandale, Frank Schmidt, Ezio Todesco, and Rob Wolf, in Proceedings of the 8th European Particle Accelerator Conference, Paris, 2002 (EPS-IGA and CERN, Geneva, 2002), pp. 260-262.

[2] Sebastien Amet, Luca Bottura, Valeria Granata, Sandrine Le Naour, Rakesh Mishra, Luc-Rene Oberli, David Richter, Stephane Sanfilippo, Arjan Verweij, Louis Walckiers, and Rob Wolf, IEEE Trans. Appl. Supercond. 13, 1239 (2003).

[3] Lucio Rossi, IEEE Trans. Appl. Supercond. 14, 153 (2004).

[4] Rob Wolf, in Proceedings to the 15th International Magnet Technology Conference, Beijing, China, 1998, pp. 238241.

[5] Laurent Deniau, in Proceedings to the LHC Project Workshop, Chamonix XIII, CERN-AB-2004-014, Chamonix, France, 2004, pp. 190-195.

[6] H. Bruck, D. Gall, J. Krzywinski, R. Lge, R. Meinke, H. Preissner, P. Schmuser, and Y. Zhao, in Proceedings of the 2nd European Particle Accelerator Conference, Nice, France, 1990 (Editions Frontières, Nice, France, 1990), pp. 1160-1162.

[7] Peter Schmueser, Rep. Prog. Phys. 54, 683 (1991).

[8] Karl Hubert Mess, Siegfried Wolff, and Peter Schmüser, Superconducting Accelerator Magnets (World Scientific Publishing, Singapore, 1996).

[9] Luca Bottura, IEEE Trans. Appl. Supercond. 10, 1054 (2000).

[10] Marco Calvi, Ezio Todesco, Luca Bottura, Stephane Sanfilippo, and Andrzej Siemko, in Proceedings of the International Conference on Magnet Technology (MT19), Genova, Italy, 2005 [IEEE Trans. Appl. Supercond. (to be published)].

[11] Dave Finley, Don Edwards, Ray Hanft, Rolland Johnson, Alfred McInturff, and Jim Strait, in Proceedings of the 
12th Particle Accelerator Conference, Washington, DC, 1987 (IEEE, Piscataway, NJ, 1987), pp. 151-153.

[12] Arjan Verweij, in Electrodynamics of Superconducting Cables in Accelerator Magnets, edited by Arjan Verweij (Universiteit Twente, Twente, Nederlands, 1995), Chap. 5, p. 101 .
[13] Ray Hanft, Bruce Brown, David Herrup, Mike Lamm, Alfred McInturff, and Mike Syphers, IEEE Trans. Magn. 25, 1647 (1989).

[14] Luca Bottura, Nicholas Sammut, Tatiana Pieloni, and Joseph Micallef, CERN LHC Project Note No. 361 (to be published). 